\title{
Genomic imprinting mechanisms in embryonic and extraembryonic mouse tissues
}

\author{
QJ Hudson, TM Kulinski, SP Huetter and DP Barlow \\ CeMM, Research Center for Molecular Medicine of the Austrian Academy of Science, Vienna Biocenter, Vienna, Austria
}

\begin{abstract}
Imprinted genes in mice and humans mainly occur in clusters that are associated with differential DNA methylation of an imprint control element (ICE) and at least one nonproteincoding RNA (ncRNA). Imprinted gene silencing is achieved by parental-specific insulator activity of the unmethylated ICE mediated by CTCF (CCCTC-binding factor) binding, or by ncRNA expression from a promoter in the unmethylated ICE. In many imprinted clusters, some genes, particularly those located furthest away from the ICE, show imprinted expression only in extraembryonic tissues. Recent research indicates that genes showing imprinted expression only in extraembryonic tissues may be regulated by different epigenetic mechanisms compared with genes showing imprinted expression in extraembryonic tissues and in
\end{abstract}

embryonic/adult tissues. The study of extraembryonic imprinted expression, thus, has the potential to illuminate novel epigenetic strategies, but is complicated by the need to collect tissue from early stages of mouse development, when extraembryonic tissues may be contaminated by maternal cells or be present in limited amounts. Research in this area would be advanced by the development of an in vitro model system in which genetic experiments could be conducted in less time and at a lower cost than with mouse models. Here, we summarize what is known about the mechanisms regulating imprinted expression in mouse extraembryonic tissues and explore the possibilities for developing an in vitro model.

Heredity (2010) 105, 45-56; doi:10.1038/hdy.2010.23; published online 17 March 2010

Keywords: genomic imprinting; placenta; yolk sac; noncoding RNA; insulator

\section{Genomic imprinting in mammals}

Both the maternal and paternal genomes are required for mammalian development, indicating that they are not equivalent and suggesting the existence of genes showing imprinted expression (Lyon and Glenister, 1977; McGrath and Solter, 1984; Surani et al., 1984; Cattanach and Kirk, 1985). This was later confirmed by the identification of imprinted genes expressed only from the maternal or paternal allele, in contrast to the majority of mammalian genes that are equally expressed or repressed from both parental alleles (Barlow et al., 1991; Bartolomei et al., 1991; DeChiara et al., 1991). Imprinted genes are now known to mainly exist in clusters in which individual genes are not autonomously imprinted, but instead imprinted expression of the whole cluster is controlled by a cis-acting genomic 'imprint'. The genomic imprint is a DNA methylation mark that is deposited on a CpG-rich DNA sequence (known as the imprint control element or ICE) during oogenesis or spermatogenesis by the DNMT3A/DNMT3L de novo methyltransferase complex (Bourc'his et al., 2001; Hata et al., 2002; Kaneda et al., 2004; Jia et al., 2007). This gametic DNA methylation mark is then maintained on the maternal or paternal allele by the maintenance DNA methyltransferase

Correspondence: Dr DP Barlow, CeMM, Research Center for Molecular Medicine of the Austrian Academy of Science, Vienna Biocenter, Dr Bohr-Gasse 9/4, Vienna 1030, Austria.

E-mail: denise.barlow@univie.ac.at

Received 30 October 2009; revised 14 January 2010; accepted 2

February 2010; published online 17 March 2010
DNMT1 (Li et al., 1993). Approximately 100 imprinted genes clustered in 25 genomic regions, have been identified in mice and humans (for details see http://www.har.mrc.ac.uk/research/genomic_imprinting/ (Williamson et al., 2009)). In spite of this small number, many imprinted genes are developmentally important and require the correct expression level (that is, one copy expressed) for normal growth and development to occur during embryogenesis.

Imprinted genes thus occur in clusters associated with the methyl-sensitive ICE that controls their imprinted expression. The ICE methylation imprint is universal, present in all tissues and at all stages of development (except germ cells), whereas imprinted expression is not always present and may vary during development, differentiation and disease. For example, the Igf2r gene is initially biallelically expressed in pre-implantation embryos, with imprinted expression beginning in postimplantation embryos (Szabo and Mann, 1995; Lerchner and Barlow, 1997). This developmental regulation is paralleled in an in vitro embryonic stem (ES) cell differentiation system (Latos et al., 2009). Imprinted expression can also be lost later during cellular differentiation in a tissue-specific manner. For example, in post-mitotic neurons, imprinted expression of $I g f 2 r$ is lost and it shows biallelic expression (Yamasaki et al., 2005). In addition, in the brain, the Ube3A gene shows imprinted expression only in the neurons, whereas in glial cells, it gains biallelic expression (Yamasaki et al., 2003). In disease states, imprinted expression can also be lost. For example, many cases of human colorectal cancer 
are associated with loss of imprinted expression that results in biallelic IGF2 expression (Kaneda and Feinberg, 2005). The examples described above involve tissue-specific gain or loss of imprinted expression for genes that show widespread or 'ubiquitous' imprinted expression in embryonic or adult tissues and in extraembryonic tissues, which comprise the short-lived placenta and membranes that support the developing embryo in utero. Most genes showing this ubiquitous pattern of imprinted expression are located close to the methyl-sensitive ICE in the cluster. Another pattern is shown by the genes in the cluster that are located further away from the ICE, which show imprinted expression only in extraembryonic tissues, but are biallelically expressed or silenced in embryo or adult soma. Genes expressed in either the embryo or the adult tissues, but showing imprinted expression only in extraembryonic tissues, have mostly been studied in the placenta, and in this review, they are referred to as showing 'placentalspecific' imprinted expression. Genes that show imprinted expression in either embryonic or adult tissues, as well as in extraembryonic tissues, will be referred to as showing 'ubiquitous' imprinted expression, although as mentioned above, such genes can show tissue-specific and developmental variation in imprinted expression. It is important to note that as the ICE DNA methylation imprint is universal (that is, present on one parental chromosome in all tissues except the germ line), the imprinting mark is present regardless of imprinted expression.

In a recent review, we described six well-studied imprinted gene clusters: the Igf2r cluster, the Kcnq1 cluster, the Pws/As cluster, the Gnas cluster, the Igf2 cluster and the Dlk cluster (Koerner et al., 2009). Of these clusters, only the Igf2r cluster, the Kcnq1 cluster and the Igf2 cluster have been shown to contain placental-specific imprinted genes and, hence, we will focus on these clusters in this review (Figure 1). In these clusters, the ubiquitously imprinted genes tend to be the 'inner' genes close to the methyl-sensitive ICE, whereas the placentalspecific genes are often the 'outer' genes found further away from the ICE (Fowden et al., 2006; Feil and Berger, 2007; Miri and Varmuza, 2009). Epigenetic processes have recently been operationally divided into three different steps: an initial 'epigenator' signal originating outside the cell nucleus, which is read by an 'epigenetic initiator', which then alters the expression state, and this new state is then maintained by 'epigenetic maintainers' (Berger et al., 2009). Imprinted gene silencing has been shown to be triggered by two different epigenetic initiators: silencing by an imprinted macro nonproteincoding RNA (ncRNA) expressed from the nonmethylated allele of the ICE in the Igf2r and Kcnq1 imprinted clusters or by insulator formation induced by CTCF (CCCTC-binding factor) binding the unmethylated ICE allele in the Igf2 cluster (Bell and Felsenfeld, 2000; Hark et al., 2000; Sleutels et al., 2002; Mancini-Dinardo et al., 2006) Notably, these studies show that within one cluster, the same epigenetic initiator silences genes showing ubiquitous or placental-specific imprinted expression.

There are two main possibilities whereby the same epigenetic initiator induces imprinted expression of more genes per cluster in the placenta compared with the embryo. Either, the epigenetic initiator acts differently in the placenta compared with the embryo or epigenetic maintainers, such as DNA methylation and
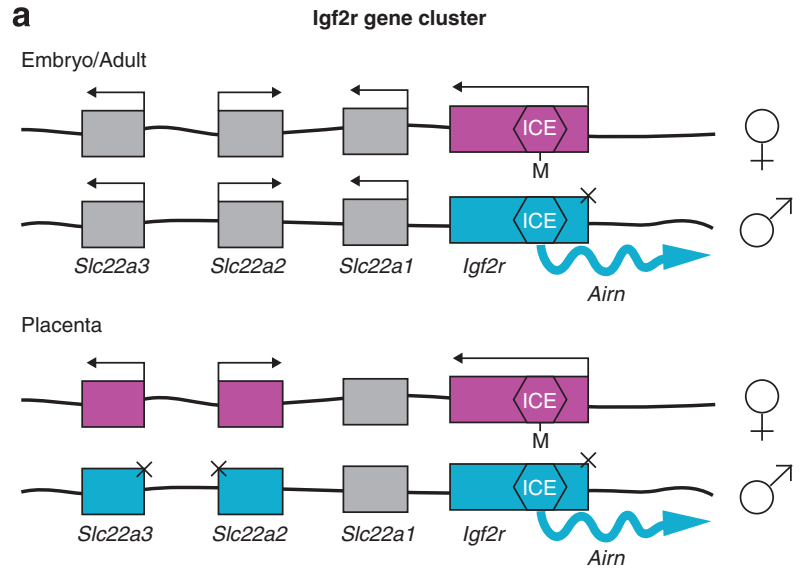

b

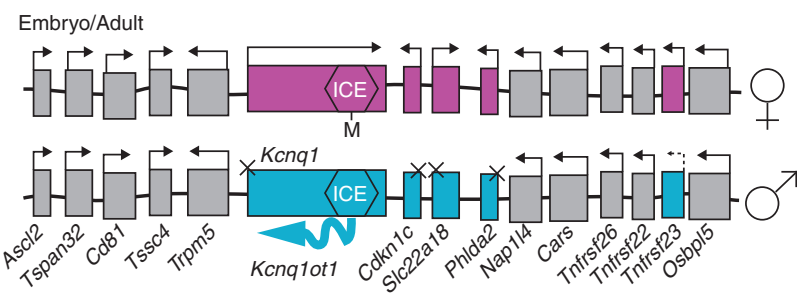

Placenta
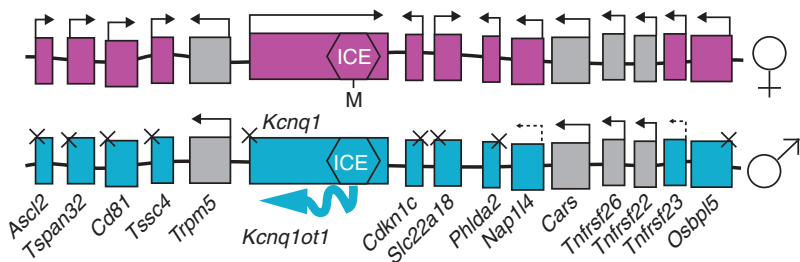

C

Igf2 gene cluster

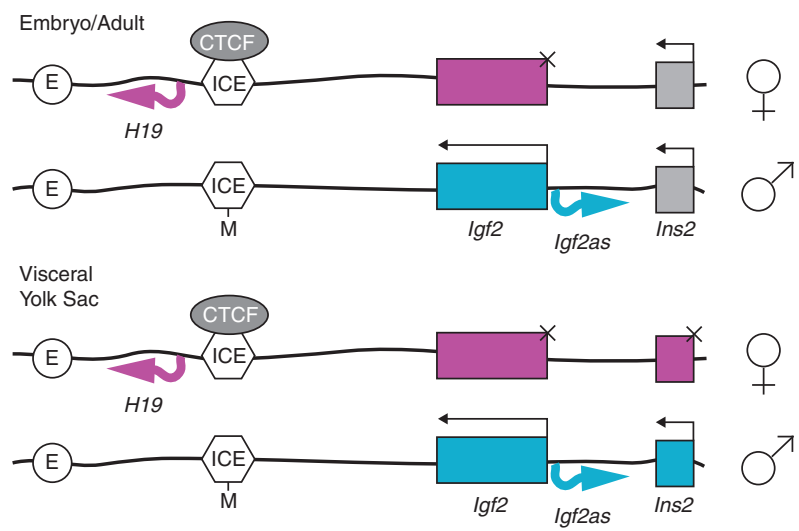

Figure 1 Three examples of mouse imprinted gene clusters showing imprinted expression in the embryo and/or adult versus placenta. (a) Igf2r cluster: imprinted silencing on the paternal chromosome is initiated by the Airn macro ncRNA. (b) Kcnq1 cluster: imprinted silencing on the paternal chromosome is initiated by the Kcnq1ot1 macro ncRNA. (c) Igf2 cluster: imprinted silencing on the maternal chromosome is initiated by CTCF binding the unmethylated ICE forming an insulator that blocks access to enhancers. Protein-coding genes and ncRNAs showing imprinted expression are shown as pink (maternally expressed) and blue (paternally expressed), with genes showing no parental allelic difference in gray. ICE, imprint control element; M, DNA methylation; and $\mathrm{E}$, enhancer. 
chromatin modifiers, could function differently in the placenta compared with the embryo. In this review, we examine the evidence for these two possibilities with reference to the developmental origin of embryonic and extraembryonic tissues.

\section{Origin of extraembryonic tissues}

The extraembryonic tissues are derived from three embryonic cell types in E4.5 pre-implantation mouse embryos, namely, the trophectoderm, the primitive endoderm and the epiblast (Figure 2). The trophectoderm differentiates only into extraembryonic lineages. In the E6.5 post-implantation embryo, trophectodermderived tissues are (i) the ectoplacental cone, which interfaces with the mother and is invaded by maternal blood, (ii) the extraembryonic ectoderm, which surrounds the upper part of the pro-amniotic cavity, and (iii) the primary trophoblast giant cells, which cover the parietal endoderm, surrounding the blastocoel (Figure 2). At E12.5, the primary trophoblast giant cells, together with the parietal endoderm, make up the parietal yolk sac, which is the outer-most extraembryonic membrane. By this stage, the ectoplacental cone and the extraembryonic ectoderm have differentiated to make up a part of the placenta, the spongiotrophoblast, the labyrinth layer and the secondary trophoblast giant cells (Figure 2) (Theiler, 1989; Rossant and Tam, 2002; Watson and Cross, 2005).

The primitive endoderm of the E4.5 embryo differentiates into parietal endoderm and visceral endoderm. The parietal endoderm forms a part of the parietal yolk sac as described above. At E6.5, the visceral endoderm can be divided into the extraembryonic visceral endoderm, which covers the extraembryonic ectoderm, and the embryonic visceral endoderm, which covers the epiblast (Figure 2). Cells from the embryonic visceral endoderm have been shown to contribute to the embryonic gut, whereas extraembryonic visceral endoderm contributes only to visceral yolk sac (VYS) in the E12.5 embryo that forms the middle extraembryonic membrane (Figure 2) (Theiler, 1989; Rossant and Tam, 2002; Kwon et al., 2008).

The third source of extraembryonic tissues is the epiblast from which the embryo proper is derived. By the E12.5 stage, epiblast-derived tissues are the visceral mesoderm, which makes up a part of the VYS, the amnion, the innermost extraembryonic membrane and the allantoic embryonic blood vessels of the placenta and the umbilical cord (Figure 2) (Theiler, 1989; Rossant and Tam, 2002; Watson and Cross, 2005). The placenta interfaces with the maternal-derived decidua and maternal blood vessels that have arterial sinuses in the labyrinth layer (Rossant and Tam, 2002; Watson and Cross, 2005) (Figure 2).

The precise site of expression of genes showing extraembryonic-specific imprinted expression is often not determined, but has been broadly characterized as placental or yolk sac specific imprinted expression (Table 1). However, for some imprinted genes, the extraembryonic cell type showing imprinted expression has been determined. For example, Ascl 2 shows imprinted expression in the trophectoderm lineage, beginning in the post-implantation embryo in the ectoplacental cone and continuing in the spongiotrophoblast of the placenta (Tanaka et al., 1999; Verhaagh et al., 2001; Lewis et al., 2006). The placental-specific imprinted gene Slc22a3 is expressed in the trophectodermderived labyrinth layer of the E12.5 placenta (Verhaagh et al., 2001). In addition to showing imprinted expression in the spongiotrophoblast of the placenta and in some embryonic tissues, Phlda2 shows imprinted expression in the primitive endoderm-derived visceral endoderm of the VYS (Frank et al., 1999). In summary, genes showing tissue-specific imprinted expression in extraembryonic tissues have been identified in cell lineages derived from the trophectoderm and primitive endoderm, but not from the epiblast.

\section{Placental-specific imprinted gene expression in well-characterized imprinted gene clusters}

The three imprinted gene clusters shown in Figure 1 show a relationship between distance from the epigenetic

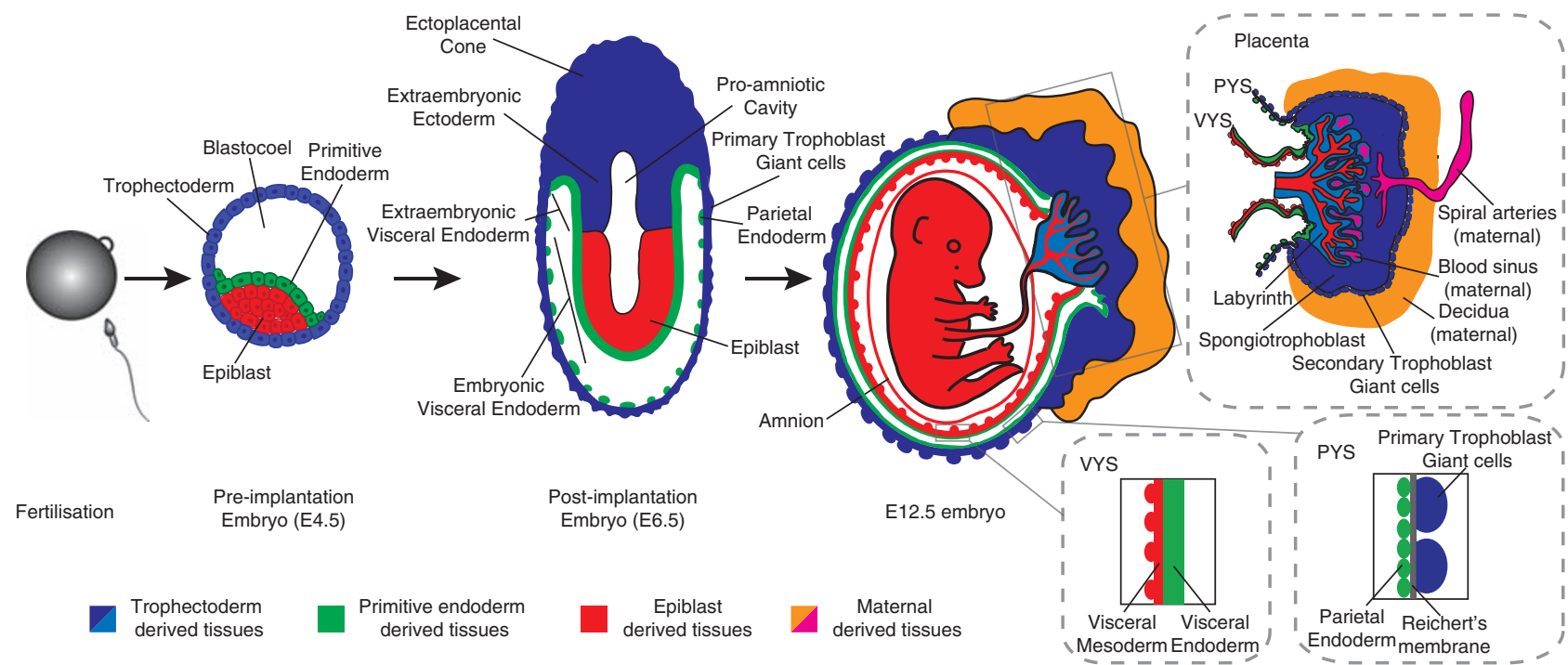

Figure 2 Development of mouse extraembryonic cell lineages. Epiblast-derived tissue is shown in red, trophectoderm-derived tissue in blue and primitive endoderm-derived tissue in green. 
Table 1 Mouse imprinted gene clusters that include genes showing imprinted expression only in extraembryonic tissues

\begin{tabular}{|c|c|c|c|c|c|}
\hline Imprinted cluster & Gene & Imprinted expression & Expressed allele & Chromosome & Reference \\
\hline \multirow[t]{7}{*}{ Peg10 } & Tfpi2 & $\mathrm{Pl}, \mathrm{YS}$ & Maternal & chr6 & (Monk et al., 2008) \\
\hline & Ppp1r9a & $\mathrm{Pl}, \mathrm{YS}$ & Maternal & chr6 & (Ono et al., 2003) \\
\hline & Pon 2 & $\mathrm{Pl}, \mathrm{YS}$ & Maternal & chr6 & (Ono et al., 2003) \\
\hline & Pon3 & $\mathrm{Pl}, \mathrm{YS}$ & Maternal & chr6 & (Ono et al., 2003) \\
\hline & Sgce & Ubiquitous & Paternal & chr6 & (Ono et al., 2003) \\
\hline & Peg10 & Ubiquitous & Paternal & chr6 & (Ono et al., 2003) \\
\hline & Asb4 & Ubiquitous & Maternal & chr6 & (Mizuno et al., 2002) \\
\hline \multirow[t]{4}{*}{ Igf2 } & $\operatorname{Ins} 2^{\mathrm{a}}$ & VYS & Paternal & chr7 & (Giddings et al., 1994; Duvillie et al., 1998) \\
\hline & $\operatorname{Igf} 2$ & Ubiquitous & Paternal & chr7 & (DeChiara et al., 1991) \\
\hline & Igf2as & Ubiquitous & Paternal & chr7 & (Rivkin et al., 1993) \\
\hline & H19 & Ubiquitous & Maternal & chr7 & (Davis et al., 1998) \\
\hline \multirow[t]{13}{*}{ Kcnq1 } & $T h$ & $\mathrm{Pl}$ & Maternal & chr7 & (Schulz et al., 2006) \\
\hline & Osbpl5 & $\mathrm{Pl}$ & Maternal & chr7 & (Engemann et al., 2000) \\
\hline & Nap1l4 & $\mathrm{Pl}$ & Maternal & chr7 & (Engemann et al., 2000) \\
\hline & $C d 81$ & Pl & Maternal & chr7 & (Caspary et al., 1998; Lewis et al., 2004b) \\
\hline & Tssc 4 & $\mathrm{Pl}$ & Maternal & chr7 & (Paulsen et al., 2000) \\
\hline & Tspan32 & Pl & Maternal & chr7 & (Umlauf et al., 2004) \\
\hline & Ascl2 & $\mathrm{Pl}$ & Maternal & chr7 & (Guillemot et al., 1995) \\
\hline & Kcnq1ot1 & Ubiquitous & Paternal & chr7 & (Smilinich et al., 1999) \\
\hline & Kcnq1 & Ubiquitous & Maternal & chr7 & (Gould and Pfeifer, 1998; Paulsen et al., 1998) \\
\hline & Cdkn1c & Ubiquitous & Maternal & chr7 & (Hatada and Mukai, 1995) \\
\hline & Slc22a18 & Ubiquitous & Maternal & chr7 & (Dao et al., 1998) \\
\hline & Phlda2 & Ubiquitous & Maternal & chr7 & $\begin{array}{l}\text { (Dunwoodie and Beddington, 2002; } \\
\text { Lewis et al. 2004b) }\end{array}$ \\
\hline & Tnfrsf23 & Ubiquitous & Maternal & chr7 & (Clark et al., 2002) \\
\hline \multirow[t]{4}{*}{ Igf2r } & Slc $22 a 2$ & $\mathrm{Pl}$ & Maternal & chr17 & (Zwart et al., 2001) \\
\hline & $S l c 22 a 3^{\mathrm{b}}$ & Pl & Maternal & chr17 & (Zwart et al., 2001) \\
\hline & $\operatorname{Ig} 2 r$ & Ubiquitous & Maternal & chr17 & (Barlow et al., 1991) \\
\hline & Airn & Ubiquitous & Paternal & chr17 & (Lyle et al., 2000) \\
\hline \multirow[t]{4}{*}{ Single imprinted genes } & Sfmbt2 & $\mathrm{Pl}, \mathrm{YS}$ & Paternal & chr2 & (Kuzmin et al., 2008) \\
\hline & Gatm & $\mathrm{Pl}, \mathrm{YS}$ & Maternal & chr2 & (Sandell et al., 2003) \\
\hline & $D c n$ & $\mathrm{Pl}$ & Maternal & chr10 & (Mizuno et al., 2002) \\
\hline & Slc38a4 & $\mathrm{Pl}$ & Paternal & $\operatorname{ch} 15$ & (Smith et al., 2003) \\
\hline
\end{tabular}

Abbreviations: Pl, placenta; VYS, visceral yolk sac; YS, yolk sacs (parietal yolk sac, visceral yolk sac and amnion not distinguished).

Genes showing imprinted gene expression in extraembryonic tissues only are shown plus other imprinted genes in their cluster that show ubiquitous imprinted expression. Ubiquitous: imprinted expression in both embryonic and extraembryonic tissues. For details see: http:// www.mousebook.org/catalog.php?catalog = imprinting (Williamson et al., 2009).

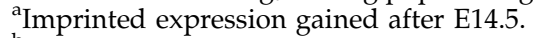

' Imprinted expression lost after E15.5.

initiator and ubiquitous or placental-specific imprinted expression. In the Igf2r and Kcnq1 imprinted gene clusters, the epigenetic initiator of imprinted gene silencing is a macro ncRNA, whose promoter lies in the ICE, whereas in the Igf2 cluster, it is the insulator function of the ICE. The imprinted genes in the three clusters show a different spatial relationship with the initiator, which may relate to how imprinted silencing occurs in these clusters.

In the Igf2r gene cluster, the paternally expressed Airn ncRNA overlaps and silences Igf2r in most tissues. In contrast Slc22a2 and Slc22a3 are not overlapped by Airn, their promoters lie 157 and $234 \mathrm{~kb}$ upstream, respectively, and they are silenced by Airn only in the placenta, but not in adult tissues (Figure 1a). Notably, Slc22a3, which lies furthest from the ICE, shows imprinted expression in early and not late placenta, whereas Slc22a2, which lies closer to the ICE, shows imprinted expression in early and late placenta (Zwart et al., 2001). On the maternal allele Igf2r, Slc22a2 and Slc22a3 are expressed because Airn is silenced by DNA methylation of the ICE (Sleutels et al., 2002; Seidl et al., 2006). In the Kcnq1 cluster, the paternally expressed Kcnq1ot1 ncRNA does not overlap with the promoter of any gene, but silences the paternal allele of the nearby genes $C d k n 1 c$, Slc22a18, Phlda2 and Kcnq1 (which contains the Kcnq1ot1 promoter and ICE in intron 10) in embryonic and extraembryonic tissues (Figure 1b) (Umlauf et al., 2004; Lewis et al., 2004a). Imprinted genes that are more distant from Kcnq1ot1, the downstream Tssc4, Cd81, Tspan32 and Ascl2 genes, as well as the upstream Osbpl5 gene, are silenced by this ncRNA only in placenta (Umlauf et al., 2004; Lewis et al., 2004a). In addition, genes internal to Osbpl5 in the cluster show partial silencing of the paternal allele Nap1l4 only in placenta and Tnfrsf 23 in both the embryo and extraembryonic tissues (Engemann et al., 2000; Clark et al., 2002). On the maternal allele, in which Kcnq1ot1 expression is silenced by DNA methylation of the ICE, all imprinted genes in the cluster are expressed. In both the Igf2r and Kcnq1 imprinted gene clusters, there are genes that do not show imprinted expression even though they are positioned closer to the ncRNA on the linear chromosome than more distant genes that do show imprinted expression (Figures 1a and b). In some cases, these genes are not expressed in tissues in which flanking genes show imprinted expression. For example, Slc22a1 is not expressed in placenta in which Slc22a2 and Slc22a3 show 
imprinted expression. However, in the Kcnq1 cluster, a number of biallelically expressed genes are present in the cluster, indicating that they are insensitive to silencing initiated by the ncRNA, whereas Nap1l4 and Tnfrsf23 that show biased expression may be only partially sensitive to ncRNA-initiated silencing.

In the Igf2 imprinted gene cluster, the ncRNAs H19 and Igf2as are located separately from the ICE and are not responsible for silencing the imprinted proteincoding genes. Instead, the ICE acts as an insulator element, with CTCF binding the nonmethylated maternal allele and silencing Igf2 by blocking access to enhancers lying downstream to the ICE and allowing these enhancers to instead promote the expression of H19 ncRNA. Methylation of the paternal allele prevents CTCF binding to the ICE, which allows the enhancers to interact with the paternal Igf2 promoter causing it to be expressed, whereas H19 is silenced (Bell and Felsenfeld, 2000; Hark et al., 2000). Igf2 shows ubiquitous imprinted expression, whereas Ins2, which lies further away from the ICE and H19, shows imprinted expression only in extraembryonic tissues, and it is unclear how imprinted expression of this gene is controlled.

Table 1 lists single imprinted genes and imprinted clusters that contain genes showing imprinted expression restricted to extraembryonic tissues. This table is incomplete because the imprinted expression pattern of many imprinted genes has not been thoroughly analyzed.

\section{Global gene regulation in extraembryonic tissues}

Extraembryonic tissues have a limited lifespan of 16 days in mouse, from setting aside of the trophectoderm linage at E3.0 to birth that occurs between E19 and 21, whereas embryonic tissues develop into adult tissues and the mouse may live for up to 2 years. Embryonic tissues also differentiate into a wider range of cell types compared with extraembryonic tissues. The differences in the function and lifespan of embryonic and extraembryonic tissues may affect the manner in which global gene regulation occurs.

Extraembryonic lineages also tolerate polyploidy more than most embryonic lineages. For example, trophoblast giant cells in the parietal yolk sac and in the placenta are polyploid. Experimentally, it has been shown that tetraploid embryos do not survive past gastrulation, whereas chimeric tetraploid/diploid embryos undergo full development and are born. In these chimeric embryos, tetraploid cells contribute mostly to extraembryonic tissues, whereas the embryo is almost entirely diploid (Tarkowski et al., 1977). The small proportion of tetraploid cells that are detected in the embryo localize to the embryonic gut and appear to be of visceral endoderm and not epiblast origin (Hadjantonakis et al., 2002; Kwon et al., 2008). This toleration of polyploid cells indicates that extraembryonic tissues are less sensitive to gene dosage than embryonic tissues, which may be a factor explaining the larger number of genes showing imprinted expression in extraembryonic tissues.

Extraembryonic tissues have been shown to have much lower levels of DNA methylation than embryonic tissues (Chapman et al., 1984; Rossant et al., 1986). This global difference in DNA methylation levels indicates that different epigenetic maintainers of gene silencing may exist in extraembryonic tissues; for example, in the absence of DNA methylation, there may be a greater role for repressive chromatin modifications in maintaining epigenetic gene silencing.

\section{Regulation of imprinted gene expression differs in extraembryonic tissues}

Many imprinted genes only show imprinted expression in extraembryonic tissues such as placenta and VYS (Table 1). The epigenetic initiator is known at the Igf2r, Kcnq1 and Igf2 imprinted clusters as described above and, importantly, the same initiator controls both ubiquitous and placental-specific imprinted expression in each cluster (Figure 1). Ubiquitous and placentalspecific imprinted gene expression could be explained by either the epigenetic initiator or epigenetic maintainers, such as DNA methylation and histone modifications, acting differentially in extraembryonic tissues. Here, we review what is known about the differences in the behavior of epigenetic initiators and maintainers of imprinted gene expression in embryonic and extraembryonic tissues and how this may affect imprinted gene silencing.

Differential maternal or paternal DNA methylation of the ICE of imprinted gene clusters is established during oogenesis or spermatogenesis, and maintained after fertilization and during development. Deletion of the unmethylated ICE allele results in loss of imprinted expression for all genes in a cluster that show either ubiquitous or placental-specific imprinted expression, in all clusters examined so far (Wutz et al., 1997; Thorvaldsen et al., 1998; Bielinska et al., 2000; Fitzpatrick et al., 2002; Lin et al., 2003; Williamson et al., 2006). DNA methylation is required for establishing and maintaining imprinted gene expression as described above. However, studies of mice lacking the maintenance DNA methyltransferase DNMT1 have shown that some genes showing placental-specific imprinted gene expression do not require DNA methylation to maintain imprinted expression. In the Kcnq1 cluster, genes showing placental-specific imprinted gene expression, Ascl2, Cd81, Osbpl5 and Tssc4, maintain imprinted expression in the absence of DNA methylation (Caspary et al., 1998; Tanaka et al., 1999; Lewis et al., 2004b). In contrast, genes closer to the ICE showing ubiquitous imprinted expression (Cdkn1c, Slc22a18, Phlda2, Kcnq1ot1), do require DNA methylation to maintain imprinted expression in both the placenta and the embryo (Lewis et al., 2004b). However, the relationship is not clear for the Kcnq1 gene, which contains the ICE within its gene body, and in the absence of DNA methylation, shows a loss of imprinted expression in the embryo, but maintains imprinted expression in the placenta (Lewis et al., 2004b). A maternal protein store of the oocyte-specific DNMT1 isoform DNMT1O transiently translocates to the nucleus at the eight-cell stage and is required for maintaining the correct methylation pattern at imprinted loci (Howell et al., 2001). Therefore, imprinted DNA methylation patterns are maintained in early DNMT1-null embryos, as the full-length nuclear-localized somatic DNMT1 isoform is not detected until the post-implantation 
stage at E7.0 (Mertineit et al., 1998). In embryos from DNMT1O-null mothers, loss of DNA methylation leads to upregulation of Kcnq1ot1 on the maternal allele, resulting in downregulation of both placental-specific and ubiquitous imprinted genes in the Kcnq1 cluster (Green et al., 2007). This shows that maintenance of DNA methylation is required in the pre-implantation embryo to maintain imprinted expression of the placentalspecific imprinted genes in the Kcnq1 cluster, but in the post-implantation embryo, it is not required for maintaining imprinted expression of these genes. Further studies are required to determine whether DNA methylation is also not required for maintenance of placentalspecific imprinted expression at other loci or whether this is a phenomenon restricted to the Kcnq1 cluster.

It has been speculated that histone modifications may have a more important role in maintaining imprinted gene expression in extraembryonic tissues than DNA methylation (Umlauf et al., 2004; Lewis et al., 2004b). At the Kcnq1 cluster, it has been shown that in the absence of DNA methylation, placental-specific imprinted genes maintain imprinted expression and the silent parental alleles are marked by the repressive histone modifications, histone $\mathrm{H} 3$ lysine 9 dimethylation (H3K9me2) and $\mathrm{H} 3$ lysine 27 trimethylation (H3K27me3) (Lewis et al., 2004b). The expressed parental alleles are marked by the active histone modifications $\mathrm{H} 3$ lysine 4 dimethylation (H3K4me2) and $\mathrm{H} 3$ acetylation of lysine 9 and 14 (H3K9ac and H3K14ac). In the embryo, these differential histone modification marks are lost on all imprinted genes except on the Kcnq1 and $C d k n 1 c$ genes that show ubiquitous imprinted expression (Umlauf et al., 2004; Lewis et al., 2004b).

The genes showing placental-specific imprinted expression in the Kcnq1 cluster are also associated with the polycomb repressive complex 2 (PRC2), responsible for adding the H3K27me3 mark to the silent allele (Umlauf et al., 2004). PRC2 is required to maintain imprinted expression of the placental-specific imprinted genes Cd81 and Tssc4 in extraembryonic tissue of E6.5 post-implantation embryos (many other placental-specific genes are not expressed at this stage) (Terranova et al., 2008). In addition, in the E6.5 embryo, PRC2 appears to be required to maintain imprinted expression of the ubiquitous imprinted gene $C d k n 1 c$ in extraembryonic tissues, but not in embryonic tissues (Terranova et al., 2008). Tfpi2, which shows placental-specific imprinted expression in the Peg10/Sgce cluster, also requires PRC2 for silencing, whereas other genes in the cluster showing ubiquitous imprinted expression do not (Monk et al., 2008). Recently, it has been shown that PRC1 is also required for silencing of genes showing placentalspecific imprinted expression in the Kcnq1 cluster. The PRC1 repressive mark H2A lysine 119 monoubiquitination $(\mathrm{H} 2 \mathrm{aK} 119 \mathrm{u} 1)$ is associated with the repressed allele of imprinted genes in undifferentiated trophoblast stem (TS) cells. In the E6.5 post-implantation embryo, PRC1 is required to maintain placental-specific imprinted expression of Cd81 and Tssc4 (Terranova et al., 2008).

The H3K9me2 methyltransferase G9A was shown to be required for genes showing placental-specific imprinted expression in the Kcnq1 cluster (Wagschal et al., 2008). Similarly, it has been shown in the Peg10/Sgce cluster that placental-specific imprinted expression of Tfpi2 requires G9A, whereas it is not required for other genes showing ubiquitous imprinted expression (Monk et al., 2008). In the Igf2r cluster, the silent allele of Slc22a3 is associated with G9A and requires G9A for imprinted expression in the placenta, whereas $I g f 2 r$ does not require G9A for imprinted expression in the embryo or placenta (Nagano et al., 2008).

These studies suggest that the repressive histone modifications H3K27me3, H2aK119u1 and H3K9me2 deposited by PRC2, PRC1 and G9A, respectively, are required for imprinted expression in the placenta. However, it remains unclear whether these repressive histone modifications are required for the initiation or maintenance of imprinted expression in the placenta, or for both.

Macro ncRNAs are associated with imprinted gene clusters and are usually expressed in a reciprocal pattern to protein-coding genes, that is, they are expressed from the parental chromosome carrying the repressed imprinted mRNA genes. These ncRNAs can be epigenetic initiators responsible for initiating imprinted gene silencing such as Kcnq1ot1 in the Kcnq1 cluster and Airn in the Igf $2 r$ cluster, or not be involved in silencing such as the H19 ncRNA in the Igf2 cluster (Sleutels et al., 2002; Mancini-Dinardo et al., 2006; Shin et al., 2008). The Kcnq1ot1 ncRNA shows imprinted expression from the two-cell embryo stage, whereas genes showing ubiquitous imprinted expression are expressed monoallelically by the blastocyst stage. However, genes showing placental-specific imprinted expression are first expressed in the ectoplacental cone of the E6.5 postimplantation embryo (Lewis et al., 2006; Terranova et al., 2008). In the $\operatorname{Ig} f 2 r$ cluster, $\operatorname{Ig} f 2 r$ is biallelically expressed in the pre-implantation embryo from the fourcell to the blastocyst stage, and then shows imprinted expression only after implantation by E6.5 (Szabo and Mann, 1995; Lerchner and Barlow, 1997). This gain of imprinted expression is paralleled in undifferentiated and differentiated ES cells, respectively (Latos et al., 2009). The onset of Airn expression in the embryo has not been determined, but it is not expressed in undifferentiated ES cells; it is paternally expressed upon differentiation together with the onset of imprinted Igf $2 r$ expression (Latos et al., 2009). In extraembryonic tissues, imprinted expression of Slc22a2 and Slc22a3 in relation to Airn ncRNA expression has not been tested before E11.5 (Zwart et al., 2001).

Truncation of the $108 \mathrm{~kb}$-long Airn and the $91 \mathrm{~kb}$-long Kcnq1ot1 to less than $3 \mathrm{~kb}$, causes loss of imprinted expression of all protein-coding genes in both embryonic and extraembryonic tissues in the Igf2r and Kcnq1 clusters, respectively (Sleutels et al., 2002; ManciniDinardo et al., 2006; Shin et al., 2008). An exception to this is the Cdkn1c gene in the Kcnq1 cluster, which retains imprinted expression in some embryonic tissues after truncation of Kcnq1ot1 (Shin et al., 2008). This indicates that either a short form of Kcnq1ot1 ncRNA is sufficient for silencing $C d k n 1 c$ in some tissues or that that two distinct mechanisms operate in this cluster. The function of macro ncRNAs in other well-studied imprinted gene clusters, such as the Pws/As, Gnas and Dlk clusters, has not yet been tested by truncation studies (Koerner et al., 2009).

The macro ncRNAs, Airn and Kcnq1ot1, silence protein-coding genes in cis, but their mechanism of action is not yet fully understood and it is unclear whether they function in the same manner to silence 
gene showing ubiquitous and placental-specific imprinted expression. An important question is whether the macro ncRNA is required at all for silencing or whether transcription itself induces silencing. These possibilities cannot be distinguished by truncation experiments, as both the RNA and transcription length are shortened. The macro ncRNA in an imprinted cluster is often positioned antisense and partially overlapping one of the imprinted genes, as is the case for $\operatorname{Igf} 2 r$, in which Airn overlaps its promoter, and Kcnq1, in which internal exons are overlapped by Kcnq1ot1. This suggests the possibility of transcriptional interference (Pauler et al., 2007), wherein transcription of the ncRNA directly inhibits transcription of the protein-coding gene, although this has not yet been shown for any imprinted cluster. For transcription of a macro ncRNA to regulate nonoverlapped genes, which includes genes showing placental-specific imprinted expression, it would have to do so indirectly, for example, by disrupting an enhancer or inducing a repressor element. Such an affect has not yet been shown at imprinted clusters. Imprinted macro ncRNAs, such as, Airn (108 kb) and Kcnq1ot1 (91 kb), are mostly unspliced and nuclear localized (Seidl et al., 2006; Pandey et al., 2008; Redrup et al., 2009). RNA interference acts in the cytoplasm to target RNA for degradation and therefore cannot be used against nuclear-localized RNA species (Zeng and Cullen, 2002). Therefore, RNA interference cannot be used to distinguish whether transcription of imprinted macro ncRNAs or the RNA product is responsible for initiating epigenetic silencing. Using an episome system, the first $5 \mathrm{~kb}$ of Kcnq1ot1 was able silence a nonoverlapped Hygromycin gene in cis, an effect that was abolished when the highly unstable c-fos $3^{\prime}$ untranslated region was inserted after the Kcnq1ot1 fragment, reducing the RNA half-life by threefold. Transcription was not affected by the c-fos $3^{\prime}$-untranslated region insertion, indicating that the RNA product was responsible for silencing (Pandey et al., 2008). Destabilization of an endogenous full-length imprinted macro ncRNA has not yet been shown, but would provide an important tool to distinguish whether imprinted silencing is mediated by ncRNA transcription or by the RNA itself (Pauler et al., 2007).

A number of studies have presented evidence for a role for RNA-mediated silencing of imprinted genes. Under this model, the imprinted ncRNA itself would target, in cis, the imprinted genes in a cluster, recruiting repressive epigenetic marks (Pauler et al., 2007). In an episome system, it has been shown that a 890-bp silencing domain in the $5^{\prime}$ region of the Kcnq1ot1 ncRNA is able to silence nonoverlapped genes in cis, implying a role for this element in the endogenous ncRNA (Mohammad et al., 2008; Pandey et al., 2008). This evidence conflicts with an in vivo truncation experiment in which the truncated Kcnq1ot1 still includes this domain, but is unable to silence imprinted genes in the Kcnq1 cluster (Mancini-Dinardo et al., 2006; Shin et al., 2008). Many imprinted macro ncRNAs contain small ncRNAs that may have a potential post-transcriptional silencing role such as microRNAs (for example, H19) and small interfering RNAs (for example, Airn) (reviewed in Koerner et al., 2009). However, using a conditional Dicer mutant, the RNA interference pathway was shown not to be involved in imprinted gene silencing at the Kcnq1 cluster in the embryo, although extraembryonic imprinted expression was not examined in this experiment (Redrup et al., 2009).

Macro ncRNAs have been suggested to recruit repressive chromatin marks to induce imprinted gene silencing (Kanduri et al., 2009). At the Kcnq1 cluster, Kcnq1ot1 has been shown to associate with a repressive chromatin domain excluding RNA polymerase II, and enriched in PRC2 and PRC1 complexes and their repressive marks H3K27me3 and H2AK119u1. The same association with a repressive compartment was also shown for Airn (Terranova et al., 2008). In the Kcnq1 cluster, Kcnq1ot1 was shown to interact with chromatin at the promoters of imprinted genes, but not with nonimprinted genes in the placenta (Pandey et al., 2008). In placenta, Airn was shown to be associated with the repressed promoter of Slc22a3, which was also associated with G9A, implicating the ncRNA in recruiting repressive histone modifications in placental-specific imprinted expression. In contrast, Airn and G9A were not associated with the promoter of $I g f 2 r$ in the placenta, a gene showing ubiquitous imprinted expression (Nagano et al., 2008). Using a Histone 3 antibody, Kcnq1ot1 was also shown to be more associated with chromatin in placenta than in fetal liver (Pandey et al., 2008). The Kcnq1ot1 RNA fluorescence in situ hybridization signal was also shown to occupy a larger volume in placenta than in embryo and to frequently overlap genes that it epigenetically silences in cis (Redrup et al., 2009). These results suggest that imprinted macro ncRNAs are involved in recruiting repressive chromatin marks to genes showing placental-specific imprinted expression to induce silencing of the repressed allele, whereas silencing of genes showing ubiquitous imprinted expression may be induced by a different downstream mechanism (Figure 3).

\section{Models explaining extraembryonic imprinted gene expression}

Models explaining the existing data on imprinted expression need to take into account the differences in imprinted gene expression between extraembryonic and embryonic tissues. In addition, different models are required to explain imprinted clusters regulated by an insulator mechanism (for example, the Igf2 cluster) and clusters regulated by a macro ncRNA (for example, the Igf2r and Kcnq1 clusters). Models to explain how imprinted gene expression could be regulated have been proposed previously (Pauler et al., 2007). On the basis of this, we propose models to specifically explain the difference between imprinted expression in embryonic and extraembryonic tissues (Figure 4).

In the Igf2 imprinted cluster, Igf2 shows ubiquitous imprinted expression, whereas Ins2 shows imprinted expression only in the VYS from E14.5 (Giddings et al., 1994; Deltour et al., 1995; Duvillie et al., 1998). It is known that silencing of Igf2 is initiated on the maternal allele by CTCF binding to the unmethylated ICE, inducing insulator function that blocks access to enhancers (Hark et al., 2000). Silencing of Ins2 could be explained by the insulator enhancer blocking function extending over a larger distance in the VYS than in the embryo (Figure 4a). How this can be achieved is unknown, but one possibility is that differences in chromatin structure 


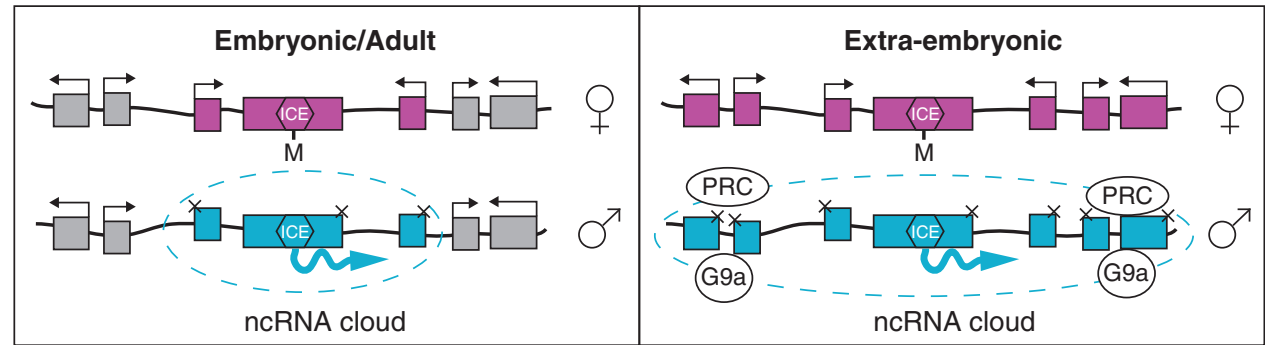

Figure 3 A summary of published work on imprinted macro ncRNA function in the embryo and in extraembryonic tissues. Genes showing imprinted expression in embryo/adult tissues tend to be close to the ICE that contains the macro ncRNA promoter. DNA methylation is important to maintain imprinted expression in the embryo. In extraembryonic tissues, genes located further away from the ICE/ncRNA promoter will show imprinted expression. DNA methylation may not be required to maintain imprinted expression of genes showing extraembryonic-specific imprinted expression, instead repressive chromatin modifications such as H3K27me3, H2AK119u1 and H3K9me2 that are deposited by polycomb complexes (PRC) and G9A are required to maintain imprinted expression. The macro ncRNA is implicated in recruiting these repressive complexes to extraembryonic-specific imprinted genes. The ncRNA forms a cloud that is larger in extraembryonic tissues and often covers the genes that are silenced (dotted blue line). ICE, imprint control element; M, DNA methylation; and E, enhancer.

and histone modifications in the Igf2-imprinted gene cluster in the VYS, compared with the embryo, could affect the range of the insulator. If such differences exist, this hypothesis could be tested by assessing the insulator function in the VYS of conditional knockouts of the relevant chromatin-modifying complexes.

Imprinted gene silencing by macro ncRNAs can be explained by transcription-mediated silencing or RNAmediated silencing models (Pauler et al., 2007). Direct interference of ncRNA transcription with the promoter of the protein-coding gene can be proposed as a silencing mechanism for genes such as Igf2r that show ubiquitous imprinted expression and in which the ncRNA Airn overlaps the promoter. Genes showing placental-specific imprinted expression tend to be further away from and not overlapped by the silencing macro ncRNA. Transcriptional interference disrupting an essential placental enhancer element may prevent upregulation of genes showing placenta-specific imprinted expression, a model that could explain, for example, imprinted expression of Slc22a2 and Slc22a3 in the Igf2r cluster (Figure 4b). In addition, transcriptional interference with an enhancer may also explain how the Kcnq1ot1 ncRNA induces ubiquitous imprinted expression of the Kcnq1 gene without overlapping its promoter. Alternatively, macro ncRNA transcription could induce a repressor element or induce formation of a repressive chromosome loop, silencing genes showing placenta-specific imprinted expression (Pauler et al., 2007).

Macro ncRNAs may also induce silencing by forming a repressive chromatin domain, recruiting repressive chromatin-modifying complexes and excluding RNA polymerase II (Terranova et al., 2008). This domain may be larger in placental tissues than in the embryo, silencing more genes (Redrup et al., 2009) (Figure 4c). Genes showing placental-specific imprinted expression have been shown to be more sensitive to silencing by repressive histone modifications than genes showing ubiquitous imprinted expression (Monk et al., 2008; Nagano et al., 2008; Terranova et al., 2008; Wagschal et al., 2008). This supports a suggestion that the mechanism by which genes showing ubiquitous imprinted expression are silenced by macro ncRNAs is different from those showing placental-specific imprinted expression.

\section{In vitro model systems for extraembryonic imprinting}

In vitro model systems of imprinted expression that mimic in vivo development provide a method to study the imprinting mechanism that is more flexible, quicker and cheaper than mouse models. The use of ES cell-based in vitro model systems that parallel some developmental stages also allows the effect of genetic modifications on imprinted expression to be tested before generating mutant mouse models. If results in such an in vitro model system indicate that a mouse is required, the already available genetically modified ES cells can be used to generate chimeras, from which mutant mice can be derived. The development of in vitro model systems of embryonic and extraembryonic imprinted gene expression is therefore a priority.

The transition from biallelic Igf2 $r$ expression in the preimplantation embryo to the onset of imprinted expression in the post-implantation embryo is paralleled by the differentiation of ES cells, providing a model for embryonic imprinted gene expression (Latos et al., 2009). A number of models of extraembryonic imprinted gene expression have been proposed (Figure 5). TS cells are stem cells derived from trophectoderm in the preimplantation embryo, a cell linage that develops into the ectoplacental cone and extraembryonic ectoderm in vivo, and then into the placental tissues that display placentalspecific imprinting (Tanaka et al., 1998; Sleutels et al., 2002; Lewis et al., 2004b, 2006; Terranova et al., 2008). In the early trophectoderm, genes that show placentalspecific imprinted expression later in development are either biallelically expressed or are not yet expressed, and in culture, TS cells that differentiate into trophoblast giant cells do not display imprinted expression of these genes (Lewis et al., 2006). Recent work has shown that TS cells can be differentiated toward a labyrinth cell fate (Hughes et al., 2004; Natale et al., 2009). In vivo, the labyrinth cell layer of the placenta shows extraembryonic-specific imprinted gene expression of genes such as Slc22a3 and Ascl2 (Tanaka et al., 1999; Verhaagh et al., 2001). Therefore, differentiation of TS cells into labyrinth cells could provide an in vitro system to study extraembryonic genomic imprinting, but validation that 


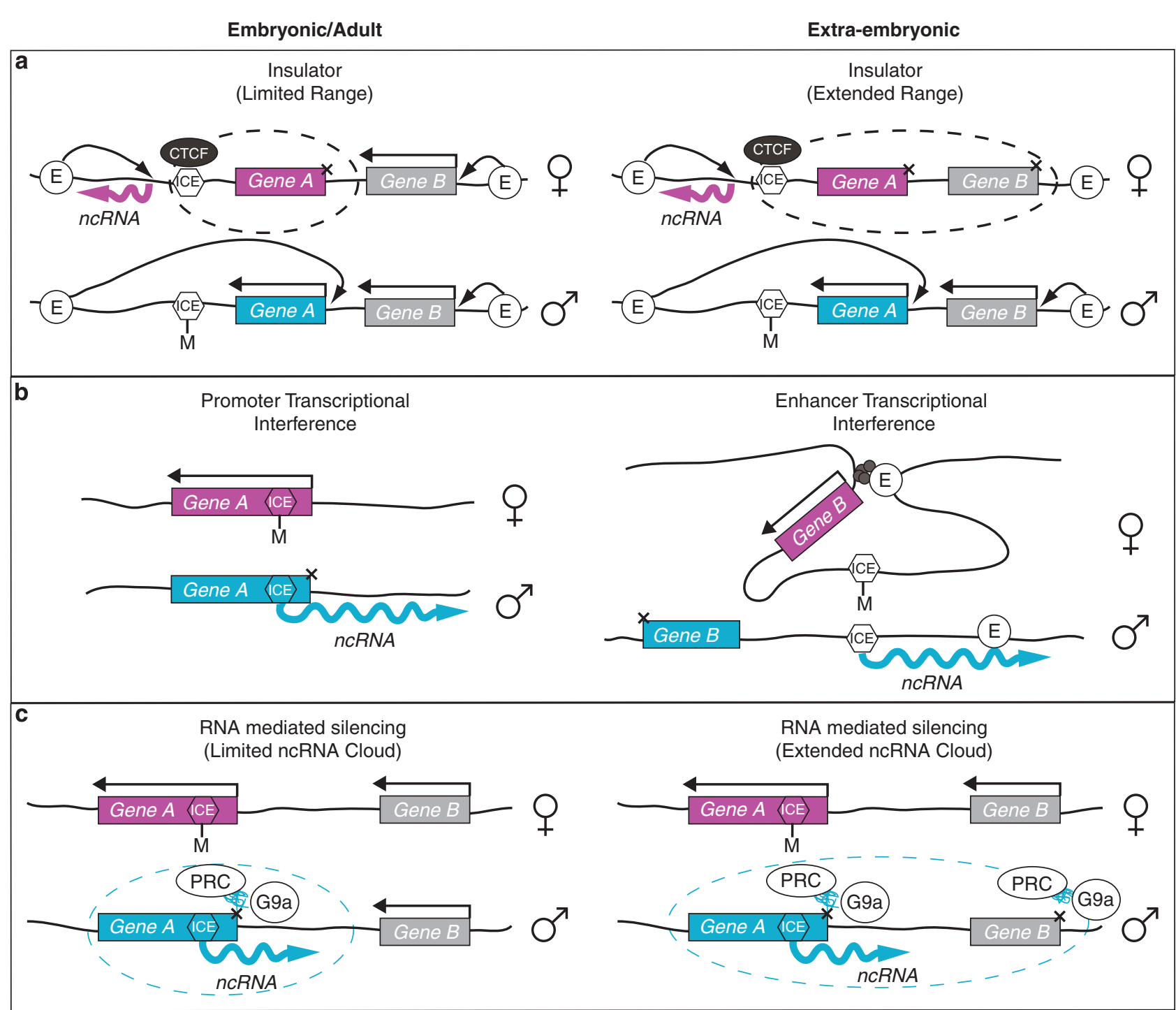

Figure 4 Models explaining embryonic and/or adult versus extraembryonic imprinted expression. (a) Insulator model: in the embryo/adult model, CTCF binds the unmethylated ICE allele and acts as an insulator, blocking access of enhancers to Gene A, whereas expression of the more distant Gene B is unaffected. In extraembryonic tissues, the insulator action extends over a greater distance also blocking enhancer access to Gene B. (b) Transcriptional interference model: in the embryo/adult model, transcription of the ncRNA overlaps the promoter of Gene A preventing upregulation of expression on this chromosome. In extraembryonic tissues, expression of the ncRNA disrupts an enhancer interaction with a distant Gene B preventing upregulation of expression on this chromosome. (c) RNA-meditated silencing model: in the embryo/adult model, the ncRNA cloud covers a limited area and the ncRNA recruits repressive chromatin-modifying complexes PRC and G9A to nearby Gene A. In extraembryonic tissues, the ncRNA cloud covers a larger area and recruits repressive chromatin modifiers to both Gene A and the more distant Gene B. ICE, imprint control element; M, DNA methylation; and E, enhancer.

placental-specific imprinted genes show imprinted expression in this system is required.

Extraembryonic endoderm (XEN) cells are stem cells derived from the blastocyst that have a cellular identity similar to primitive endoderm (Kunath et al., 2005). In vivo primitive endoderm differentiates into tissues, including the visceral endoderm that becomes part of the VYS and can show extraembryonic-specific imprinted expression for a number of genes (Table 1). However, it has not been shown that XEN cells can differentiate into yolk sac visceral endoderm in culture; hence, using currently known culture conditions, XEN cells cannot be used as a model of extraembryonic imprinted gene expression.
Embryonic stem cells cultured in leukemia inhibitory factor-free media on nonadhesive surfaces form aggregates and differentiate into structures known as embryoid bodies (EBs), which initially most closely resemble early post-implantation embryos with an outer endoderm layer and inner ectoderm layer (Doetschman et al., 1985). Following prolonged differentiation of EBs, multiple differentiated cell types can arise, including cardiomyocytes, endothelial cells, adipocytes, neuronal cells and hematopoietic cells (Desbaillets et al., 2000). It has been reported by a number of studies that EBs can differentiate into VYS-like structures expressing visceral endoderm markers (Doetschman et al., 1985; Abe et al., 1996; Koike et al., 2007). As VYS is known to express 


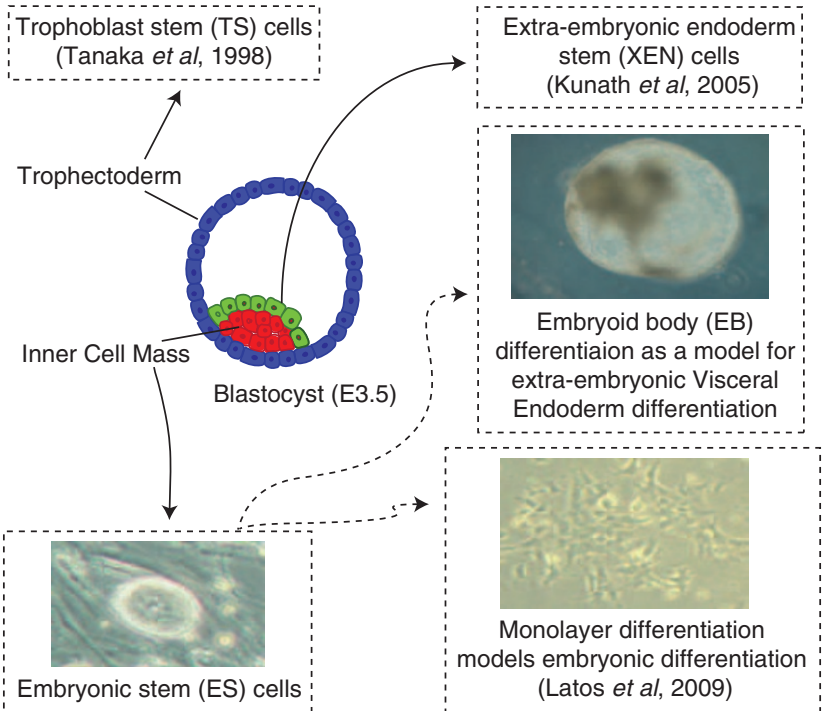

Figure 5 In vitro models to study the imprinting mechanism. ES cells derived from the ICM can be differentiated in vitro providing a model of embryonic imprinted expression. Models of extraembryonic imprinted expression are TS cells derived from trophectoderm and XEN cells derived from primitive endoderm. Differentiation of ES cells into embryoid bodies (EBs) can form tissues expressing markers of visceral endoderm and resembling VYS, suggesting that this system may provide a model of extraembryonic imprinted expression.

genes showing extraembryonic-specific imprinted expression, a robust EB culture system for deriving VYSlike visceral endoderm tissue could provide an in vitro model for extraembryonic imprinting and therefore warrants further investigation.

\section{Conclusions}

Imprinted gene expression is controlled by differential DNA methylation of an ICE, which is read by an epigenetic initiator that silences genes in the surrounding imprinted gene cluster in cis. Imprinted genes close to the epigenetic initiator tend to show ubiquitous imprinted expression, whereas genes further away can show placental-specific imprinted expression. Evidence so far indicates that, compared with genes showing ubiquitous imprinted expression, genes showing placental-specific imprinted expression are less sensitive to DNA methylation; instead, repressive histone modifications seem to have a more important role in imprinted gene expression in the placenta. An in vitro model system for extraembryonic imprinted expression would complement in vivo studies, but currently, a good model is lacking.

\section{Conflict of interest}

The authors declare no conflict of interest.

\section{Acknowledgements}

We are supported by the EU-FW6 IP 'HEROIC' (LSHGCT-2005-018883) the NoE 'The Epigenome' (LSHG-CT2004-053433) and the FWF SFB F17 Modulators of RNA Fate and Function (SFB-P1718 B10). We thank Florian
M Pauler, Martha V Koerner, Ru Huang and Jennifer L Boots for comments on the paper.

\section{References}

Abe K, Niwa H, Iwase K, Takiguchi M, Mori M, Abe SI et al. (1996). Endoderm-specific gene expression in embryonic stem cells differentiated to embryoid bodies. Exp Cell Res 229: 27-34.

Barlow DP, Stoger R, Herrmann BG, Saito K, Schweifer N (1991). The mouse insulin-like growth factor type-2 receptor is imprinted and closely linked to the Tme locus. Nature 349: 84-87.

Bartolomei MS, Zemel S, Tilghman SM (1991). Parental imprinting of the mouse H19 gene. Nature 351: 153-155.

Bell AC, Felsenfeld G (2000). Methylation of a CTCF-dependent boundary controls imprinted expression of the Igf2 gene. Nature 405: 482-485.

Berger SL, Kouzarides T, Shiekhattar R, Shilatifard A (2009). An operational definition of epigenetics. Genes Dev 23: 781-783.

Bielinska B, Blaydes SM, Buiting K, Yang T, Krajewska-Walasek M, Horsthemke B et al. (2000). De novo deletions of SNRPN exon 1 in early human and mouse embryos result in a paternal to maternal imprint switch. Nat Genet 25: 74-78.

Bourc'his D, Xu GL, Lin CS, Bollman B, Bestor TH (2001). Dnmt3L and the establishment of maternal genomic imprints. Science 294: 2536-2539.

Caspary T, Cleary MA, Baker CC, Guan XJ, Tilghman SM (1998). Multiple mechanisms regulate imprinting of the mouse distal chromosome 7 gene cluster. Mol Cell Biol 18: 3466-3474.

Cattanach BM, Kirk M (1985). Differential activity of maternally and paternally derived chromosome regions in mice. Nature 315: 496-498.

Chapman V, Forrester L, Sanford J, Hastie N, Rossant J (1984). Cell lineage-specific undermethylation of mouse repetitive DNA. Nature 307: 284-286.

Clark L, Wei M, Cattoretti G, Mendelsohn C, Tycko B (2002). The Tnfrh1 (Tnfrsf23) gene is weakly imprinted in several organs and expressed at the trophoblast-decidua interface. BMC Genet 3: 11.

Dao D, Frank D, Qian N, O'Keefe D, Vosatka RJ, Walsh CP et al. (1998). IMPT1, an imprinted gene similar to polyspecific transporter and multi-drug resistance genes. Hum Mol Genet 7: 597-608.

Davis TL, Tremblay KD, Bartolomei MS (1998). Imprinted expression and methylation of the mouse H19 gene are conserved in extraembryonic lineages. Dev Genet 23: 111-118.

DeChiara TM, Robertson EJ, Efstratiadis A (1991). Parental imprinting of the mouse insulin-like growth factor II gene. Cell 64: 849-859.

Deltour L, Montagutelli X, Guenet JL, Jami J, Paldi A (1995). Tissue- and developmental stage-specific imprinting of the mouse proinsulin gene, Ins2. Dev Biol 168: 686-688.

Desbaillets I, Ziegler U, Groscurth P, Gassmann M (2000). Embryoid bodies: an in vitro model of mouse embryogenesis. Exp Physiol 85: 645-651.

Doetschman TC, Eistetter H, Katz M, Schmidt W, Kemler R (1985). The in vitro development of blastocyst-derived embryonic stem cell lines: formation of visceral yolk sac, blood islands and myocardium. J Embryol Exp Morphol 87: 27-45.

Dunwoodie SL, Beddington RS (2002). The expression of the imprinted gene Ipl is restricted to extra-embryonic tissues and embryonic lateral mesoderm during early mouse development. Int J Dev Biol 46: 459-466.

Duvillie B, Bucchini D, Tang T, Jami J, Paldi A (1998). Imprinting at the mouse Ins2 locus: evidence for cis- and trans-allelic interactions. Genomics 47: 52-57.

Engemann S, Strodicke M, Paulsen M, Franck O, Reinhardt R, Lane $N$ et al. (2000). Sequence and functional comparison in 
the Beckwith-Wiedemann region: implications for a novel imprinting centre and extended imprinting. Hum Mol Genet 9: 2691-2706.

Feil R, Berger F (2007). Convergent evolution of genomic imprinting in plants and mammals. Trends Genet 23: 192-199.

Fitzpatrick GV, Soloway PD, Higgins MJ (2002). Regional loss of imprinting and growth deficiency in mice with a targeted deletion of KvDMR1. Nat Genet 32: 426-431.

Fowden AL, Sibley C, Reik W, Constancia M (2006). Imprinted genes, placental development and fetal growth. Horm Res 65 (Suppl 3): 50-58.

Frank D, Mendelsohn CL, Ciccone E, Svensson K, Ohlsson R, Tycko B (1999). A novel pleckstrin homology-related gene family defined by Ipl/Tssc3, TDAG51, and Tih1: tissuespecific expression, chromosomal location, and parental imprinting. Mamm Genome 10: 1150-1159.

Giddings SJ, King CD, Harman KW, Flood JF, Carnaghi LR (1994). Allele specific inactivation of insulin 1 and 2, in the mouse yolk sac, indicates imprinting. Nat Genet 6: 310-313.

Gould TD, Pfeifer K (1998). Imprinting of mouse Kvlqt1 is developmentally regulated. Hum Mol Genet 7: 483-487.

Green K, Lewis A, Dawson C, Dean W, Reinhart B, Chaillet JR et al. (2007). A developmental window of opportunity for imprinted gene silencing mediated by DNA methylation and the Kcnq1ot1 noncoding RNA. Mamm Genome 18: 32-42.

Guillemot F, Caspary T, Tilghman SM, Copeland NG, Gilbert DJ, Jenkins NA et al. (1995). Genomic imprinting of Mash2, a mouse gene required for trophoblast development. Nat Genet 9: 235-242.

Hadjantonakis AK, Macmaster S, Nagy A (2002). Embryonic stem cells and mice expressing different GFP variants for multiple non-invasive reporter usage within a single animal. BMC Biotechnol 2: 11.

Hark AT, Schoenherr CJ, Katz DJ, Ingram RS, Levorse JM, Tilghman SM (2000). CTCF mediates methylation-sensitive enhancer-blocking activity at the H19/Igf2 locus. Nature 405: 486-489.

Hata K, Okano M, Lei H, Li E (2002). Dnmt3L cooperates with the Dnmt3 family of de novo DNA methyltransferases to establish maternal imprints in mice. Development 129: 1983-1993.

Hatada I, Mukai T (1995). Genomic imprinting of p57KIP2, a cyclin-dependent kinase inhibitor, in mouse. Nat Genet 11: 204-206.

Howell CY, Bestor TH, Ding F, Latham KE, Mertineit C, Trasler JM et al. (2001). Genomic imprinting disrupted by a maternal effect mutation in the Dnmt1 gene. Cell 104: 829-838.

Hughes M, Dobric N, Scott IC, Su L, Starovic M, St-Pierre B et al. (2004). The Hand1, Stra13 and Gcm1 transcription factors override FGF signaling to promote terminal differentiation of trophoblast stem cells. Dev Biol 271: 26-37.

Jia D, Jurkowska RZ, Zhang X, Jeltsch A, Cheng X (2007). Structure of Dnmt3a bound to Dnmt3L suggests a model for de novo DNA methylation. Nature 449: 248-251.

Kanduri C, Whitehead J, Mohammad F (2009). The long and the short of it: RNA-directed chromatin asymmetry in mammalian X-chromosome inactivation. FEBS Lett 583: 857-864.

Kaneda A, Feinberg AP (2005). Loss of imprinting of IGF2: a common epigenetic modifier of intestinal tumor risk. Cancer Res 65: 11236-11240.

Kaneda M, Okano M, Hata K, Sado T, Tsujimoto N, Li E et al. (2004). Essential role for de novo DNA methyltransferase Dnmt3a in paternal and maternal imprinting. Nature 429: 900-903.

Koerner MV, Pauler FM, Huang R, Barlow DP (2009). The function of non-coding RNAs in genomic imprinting. Development 136: 1771-1783.

Koike M, Sakaki S, Amano Y, Kurosawa H (2007). Characterization of embryoid bodies of mouse embryonic stem cells formed under various culture conditions and estimation of differentiation status of such bodies. I Biosci Bioeng 104: 294-299.

Kunath T, Arnaud D, Uy GD, Okamoto I, Chureau C, Yamanaka $Y$ et al. (2005). Imprinted X-inactivation in extra-embryonic endoderm cell lines from mouse blastocysts. Development 132 1649-1661.

Kuzmin A, Han Z, Golding MC, Mann MR, Latham KE, Varmuza S (2008). The PcG gene Sfmbt2 is paternally expressed in extraembryonic tissues. Gene Expr Patterns 8: 107-116.

Kwon GS, Viotti M, Hadjantonakis AK (2008). The endoderm of the mouse embryo arises by dynamic widespread intercalation of embryonic and extraembryonic lineages. Dev Cell 15: 509-520.

Latos PA, Stricker SH, Steenpass L, Pauler FM, Huang R, Senergin BH et al. (2009). An in vitro ES cell imprinting model shows that imprinted expression of the Igf2r gene arises from an allele-specific expression bias. Development 136: 437-448.

Lerchner W, Barlow DP (1997). Paternal repression of the imprinted mouse Igf $2 \mathrm{r}$ locus occurs during implantation and is stable in all tissues of the post-implantation mouse embryo. Mech Dev 61: 141-149.

Lewis A, Green K, Dawson C, Redrup L, Huynh KD, Lee JT et al. (2006). Epigenetic dynamics of the Kcnq1 imprinted domain in the early embryo. Development 133: 4203-4210.

Lewis A, Mitsuya K, Constancia M, Reik W (2004a). Tandem repeat hypothesis in imprinting: deletion of a conserved direct repeat element upstream of $\mathrm{H} 19$ has no effect on imprinting in the Igf2-H19 region. Mol Cell Biol 24: 5650-5656.

Lewis A, Mitsuya K, Umlauf D, Smith P, Dean W, Walter J et al. (2004b). Imprinting on distal chromosome 7 in the placenta involves repressive histone methylation independent of DNA methylation. Nat Genet 36: 1291-1295.

Li E, Beard C, Jaenisch R (1993). Role for DNA methylation in genomic imprinting. Nature 366: 362-365.

Lin SP, Youngson N, Takada S, Seitz H, Reik W, Paulsen M et al. (2003). Asymmetric regulation of imprinting on the maternal and paternal chromosomes at the Dlk1-Gtl2 imprinted cluster on mouse chromosome 12. Nat Genet 35: 97-102.

Lyle R, Watanabe D, te Vruchte D, Lerchner W, Smrzka OW, Wutz A et al. (2000). The imprinted antisense RNA at the Igf2r locus overlaps but does not imprint Mas1. Nat Genet 25: $19-21$.

Lyon MF, Glenister PH (1977). Factors affecting the observed number of young resulting from adjacent-2 disjunction in mice carrying a translocation. Genet Res 29: 83-92.

Mancini-Dinardo D, Steele SJ, Levorse JM, Ingram RS, Tilghman SM (2006). Elongation of the Kcnq1ot1 transcript is required for genomic imprinting of neighboring genes. Genes Dev 20: $1268-1282$

McGrath J, Solter D (1984). Completion of mouse embryogenesis requires both the maternal and paternal genomes. Cell 37: $179-183$.

Mertineit C, Yoder JA, Taketo T, Laird DW, Trasler JM, Bestor TH (1998). Sex-specific exons control DNA methyltransferase in mammalian germ cells. Development 125: 889-897.

Miri K, Varmuza S (2009). Imprinting and extraembryonic tissues-mom takes control. Int Rev Cell Mol Biol 276: 215-262.

Mizuno Y, Sotomaru Y, Katsuzawa Y, Kono T, Meguro M, Oshimura $\mathrm{M}$ et al. (2002). Asb4, Ata3, and Dcn are novel imprinted genes identified by high-throughput screening using RIKEN cDNA microarray. Biochem Biophys Res Commun 290: 1499-1505.

Mohammad F, Pandey RR, Nagano T, Chakalova L, Mondal T, Fraser $\mathrm{P}$ et al. (2008). Kcnq1ot1/Lit1 noncoding RNA mediates transcriptional silencing by targeting to the perinucleolar region. Mol Cell Biol 28: 3713-3728.

Monk D, Wagschal A, Arnaud P, Muller PS, Parker-Katiraee L, Bourc'his D et al. (2008). Comparative analysis of human 
chromosome 7q21 and mouse proximal chromosome 6 reveals a placental-specific imprinted gene, TFPI2/Tfpi2, which requires EHMT2 and EED for allelic-silencing. Genome Res 18: 1270-1281.

Nagano T, Mitchell JA, Sanz LA, Pauler FM, Ferguson-Smith AC, Feil R et al. (2008). The air noncoding RNA epigenetically silences transcription by targeting G9a to chromatin. Science 322: $1717-1720$.

Natale DR, Hemberger M, Hughes M, Cross JC (2009). Activin promotes differentiation of cultured mouse trophoblast stem cells towards a labyrinth cell fate. Dev Biol 335: 120-131.

Ono R, Shiura H, Aburatani H, Kohda T, Kaneko-Ishino T, Ishino $F$ (2003). Identification of a large novel imprinted gene cluster on mouse proximal chromosome 6. Genome Res 13: 1696-1705.

Pandey RR, Mondal T, Mohammad F, Enroth S, Redrup L, Komorowski J et al. (2008). Kcnq1ot1 antisense noncoding RNA mediates lineage-specific transcriptional silencing through chromatin-level regulation. Mol Cell 32: 232-246.

Pauler FM, Koerner MV, Barlow DP (2007). Silencing by imprinted noncoding RNAs: is transcription the answer? Trends Genet 23: 284-292.

Paulsen M, Davies KR, Bowden LM, Villar AJ, Franck O, Fuermann M et al. (1998). Syntenic organization of the mouse distal chromosome 7 imprinting cluster and the BeckwithWiedemann syndrome region in chromosome 11p15.5. Hum Mol Genet 7: 1149-1159.

Paulsen M, El-Maarri O, Engemann S, Strodicke M, Franck O, Davies K et al. (2000). Sequence conservation and variability of imprinting in the Beckwith-Wiedemann syndrome gene cluster in human and mouse. Hum Mol Genet 9: 1829-1841.

Redrup L, Branco MR, Perdeaux ER, Krueger C, Lewis A, Santos F et al. (2009). The long noncoding RNA Kcnq1ot1 organises a lineage-specific nuclear domain for epigenetic gene silencing. Development 136: 525-530.

Rivkin M, Rosen KM, Villa-Komaroff L (1993). Identification of an antisense transcript from the IGF-II locus in mouse. Mol Reprod Dev 35: 394-397.

Rossant J, Sanford JP, Chapman VM, Andrews GK (1986). Undermethylation of structural gene sequences in extraembryonic lineages of the mouse. Dev Biol 117: 567-573.

Rossant J, Tam PPL (2002). Mouse Development: Patterning, Morphogenesis, and Organogenesis. Academic Press: San Diego.

Sandell LL, Guan XJ, Ingram R, Tilghman SM (2003). Gatm, a creatine synthesis enzyme, is imprinted in mouse placenta. Proc Natl Acad Sci USA 100: 4622-4627.

Schulz R, Menheniott TR, Woodfine K, Wood AJ, Choi JD, Oakey RJ (2006). Chromosome-wide identification of novel imprinted genes using microarrays and uniparental disomies. Nucleic Acids Res 34: e88.

Seidl CI, Stricker SH, Barlow DP (2006). The imprinted Air ncRNA is an atypical RNAPII transcript that evades splicing and escapes nuclear export. EMBO J 25: 3565-3575.

Shin JY, Fitzpatrick GV, Higgins MJ (2008). Two distinct mechanisms of silencing by the KvDMR1 imprinting control region. $E M B O$ J 27: 168-178.

Sleutels F, Zwart R, Barlow DP (2002). The non-coding Air RNA is required for silencing autosomal imprinted genes. Nature 415: 810-813.

Smilinich NJ, Day CD, Fitzpatrick GV, Caldwell GM, Lossie AC, Cooper PR et al. (1999). A maternally methylated CpG island in KvLQT1 is associated with an antisense paternal transcript and loss of imprinting in Beckwith-Wiedemann syndrome. Proc Natl Acad Sci USA 96: 8064-8069.

Smith RJ, Dean W, Konfortova G, Kelsey G (2003). Identification of novel imprinted genes in a genome-wide screen for maternal methylation. Genome Res 13: 558-569.
Surani MA, Barton SC, Norris ML (1984). Development of reconstituted mouse eggs suggests imprinting of the genome during gametogenesis. Nature 308: 548-550.

Szabo PE, Mann JR (1995). Allele-specific expression and total expression levels of imprinted genes during early mouse development: implications for imprinting mechanisms. Genes Dev 9: 3097-3108

Tanaka M, Puchyr M, Gertsenstein M, Harpal K, Jaenisch R, Rossant J et al. (1999). Parental origin-specific expression of Mash2 is established at the time of implantation with its imprinting mechanism highly resistant to genome-wide demethylation. Mech Dev 87: 129-142.

Tanaka S, Kunath T, Hadjantonakis AK, Nagy A, Rossant J (1998). Promotion of trophoblast stem cell proliferation by FGF4. Science 282: 2072-2075.

Tarkowski AK, Witkowska A, Opas J (1977). Development of cytochalasin in B-induced tetraploid and diploid/tetraploid mosaic mouse embryos. J Embryol Exp Morphol 41: 47-64.

Terranova R, Yokobayashi S, Stadler MB, Otte AP, van Lohuizen M, Orkin SH et al. (2008). Polycomb group proteins Ezh2 and Rnf2 direct genomic contraction and imprinted repression in early mouse embryos. Dev Cell 15: 668-679.

Theiler K (1989). The House Mouse-Atlas of Embryonic Development. Springer-Verlag: New York.

Thorvaldsen JL, Duran KL, Bartolomei MS (1998). Deletion of the H19 differentially methylated domain results in loss of imprinted expression of H19 and Igf2. Genes Dev 12: 3693-3702.

Umlauf D, Goto Y, Cao R, Cerqueira F, Wagschal A, Zhang Y et al. (2004). Imprinting along the Kcnq1 domain on mouse chromosome 7 involves repressive histone methylation and recruitment of Polycomb group complexes. Nat Genet 36: 1296-1300.

Verhaagh S, Barlow DP, Zwart R (2001). The extraneuronal monoamine transporter Slc22a3/Orct3 co-localizes with the Maoa metabolizing enzyme in mouse placenta. Mech Dev 100: $127-130$.

Wagschal A, Sutherland HG, Woodfine K, Henckel A, Chebli K, Schulz R et al. (2008). G9a histone methyltransferase contributes to imprinting in the mouse placenta. Mol Cell Biol 28: 1104-1113.

Watson ED, Cross JC (2005). Development of structures and transport functions in the mouse placenta. Physiology (Bethesda) 20: 180-193.

Williamson CM, Blake A, Thomas S, Beechey CV, Hancock J, Cattanach BM et al. (2009). World Wide Web Site-Mouse Imprinting Data and References. MRC Harwell: Oxfordshire. http://www.har.mrc.ac.uk/research/genomic_imprinting/.

Williamson CM, Turner MD, Ball ST, Nottingham WT, Glenister P, Fray M et al. (2006). Identification of an imprinting control region affecting the expression of all transcripts in the GNAS cluster. Nat Genet 38: 350-355.

Wutz A, Smrzka OW, Schweifer N, Schellander K, Wagner EF, Barlow DP (1997). Imprinted expression of the Igf2r gene depends on an intronic CpG island. Nature 389: 745-749.

Yamasaki K, Joh K, Ohta T, Masuzaki H, Ishimaru T, Mukai T et al. (2003). Neurons but not glial cells show reciprocal imprinting of sense and antisense transcripts of Ube3a. Hum Mol Genet 12: 837-847.

Yamasaki Y, Kayashima T, Soejima H, Kinoshita A, Yoshiura K, Matsumoto $\mathrm{N}$ et al. (2005). Neuron-specific relaxation of Igf2r imprinting is associated with neuron-specific histone modifications and lack of its antisense transcript Air. Hum Mol Genet 14: 2511-2520.

Zeng Y, Cullen BR (2002). RNA interference in human cells is restricted to the cytoplasm. RNA 8: 855-860.

Zwart R, Sleutels F, Wutz A, Schinkel AH, Barlow DP (2001). Bidirectional action of the Igf2r imprint control element on upstream and downstream imprinted genes. Genes Dev 15: 2361-2366 